\title{
REMARKS ON PETTIS INTEGRABILITY
}

\author{
R. HUFF
}

ABstract. Characterizations of Pettis integrability, including the GeitzTalagrand core theorem, are derived in an easy way.

The purpose of this note is to point out how a folklore result (Proposition 1) can be made the basis for relatively easy proofs of some recent results about Pettis integrability. Our notation follows Dunford and Schwartz [1].

Let $(\Omega, \Sigma, \lambda)$ be a complete probability space, and let $X$ be a Banach space with continuous dual $X^{*}$. A function $f: \Omega \rightarrow X$ is Dunford integrable provided the composition $T\left(x^{*}\right)=x^{*} f$ is in $L^{1}(\lambda)$ for every $x^{*}$ in $X^{*}$. In this case, it follows (from the closed graph theorem) that $T: X^{*} \rightarrow L^{1}(\lambda)$ is a bounded linear operator. Hence, for every $g$ in $L^{\infty}(\lambda)$, the map $\varphi_{g}$, defined by

$$
\varphi_{g}\left(x^{*}\right)=\int g T\left(x^{*}\right) d \lambda,
$$

is in $X^{* *}$. In particular, for each $E$ in $\Sigma, \nu(E)=\int_{E} f d \lambda$, defined to equal $\varphi_{\chi_{E}}$ and called the Dunford integral of $f$ over $E$, is an element of $X^{* *}$.

The function $\nu: \Sigma \rightarrow X^{* *}$ is not necessarily countably additive. It can be shown that $\nu$ is countably additive if and only if $T$ is a weakly compact operator if and only if $\left\{x^{*} f:\left\|x^{*}\right\| \leq 1\right\}$ is uniformly integrable in $L^{1}(\lambda)[\mathbf{1}$, pp. 319, 485, 292]. These conditions are automatically satisfied if $f$ has bounded range.

Let $\hat{X}$ denote the natural image of $X$ in $X^{* *}$. The function $f$ is said to be Pettis integrable if and only if for every $E$ in $\Sigma, \nu(E)$ is in $\hat{X}$ (equivalently, $\nu(E)$ is weak* continuous on $\left.X^{*}\right)$. The following proposition is essentially a reformulation of the definition.

Proposition 1. A Dunford integrable function $f$ is Pettis integrable if and only if the operator $T: X^{*} \rightarrow L^{1}(\lambda)$ is weak ${ }^{*}$-to-weak continuous.

In particular, if $f$ is Pettis integrable then $T$ is necessarily a weakly compact operator.

ProOF. $(\Leftarrow)$ is clear.

$(\Rightarrow)$ If $f$ is Pettis integrable, then for each simple function $g$ in $L^{\infty}(\lambda), \varphi_{g}$ is weak* continuous on $X^{*}$. By approximation, $\varphi_{g}$ is weak* continuous for every $g$ in $L^{\infty}(\lambda)$.

Therefore, to study Pettis integrability one studies the action of $T$ on weak* neighborhoods in $X^{*}$. If $F$ is a finite set in $X$, and $\varepsilon>0$, let

$$
\mathcal{K}(F, \varepsilon)=\left\{x^{*} \in X^{*}:\left\|x^{*}\right\| \leq 1 \text { and } x^{*}(x) \leq \varepsilon \text { for every } x \text { in } F\right\} .
$$

Received by the editors March 9, 1985.

1980 Mathematics Subject Classification. Primary 28B05, 46G10; Secondary 46 B99. 
Lemma 2. If $f$ is Dunford integrable, then for all $F, \varepsilon$ the set $T(K(F, \varepsilon))$ is closed and convex in $L^{1}(\lambda)$.

Proof. Convexity is clear. Suppose $g$ is in the closure of $T(K(F, \varepsilon))$, and choose $x_{n}^{*}$ in $\mathcal{K}(F, \varepsilon)$ with $x_{n}^{*} f \rightarrow g$ a.e. Let $x^{*}$ be a weak ${ }^{*}$ cluster point of $\left(x_{n}^{*}\right)_{n}$. Then $x^{*}$ is in $K(F, \varepsilon)$ and $g=x^{*} f$ a.e.

The following reformulation of Proposition 1 was derived from ideas in proofs due to M. Talagrand (see Sentilles and Wheeler [5]).

Proposition 3. If $f$ is Dunford integrable, then the following are equivalent:

1. $f$ is Pettis integrable;

2. $T$ is a weakly compact operator and

$$
\{0\}=\bigcap\{T(K(F, \varepsilon)) \mid F \subset X, F \text { finite, and } \varepsilon>0\} .
$$

Proof. (1) $\Rightarrow(2)$ If $f$ is Pettis integrable, then $T$ is weakly compact. Suppose $g$ is in $\bigcap_{(F, \varepsilon)} T(\mathcal{K}(F, \varepsilon))$. For each $(F, \varepsilon)$ choose $x_{F, \varepsilon}^{*}$ in $\mathcal{K}(F, \varepsilon)$ so that $g=T\left(x_{F, \varepsilon}^{*}\right)$. Note that $\left(x_{F, \varepsilon}^{*}\right)_{(F, \varepsilon)}$ is naturally a net in $X^{*}$ which converges weak ${ }^{*}$ to 0 . Hence, $g=T\left(x_{F, \varepsilon}^{*}\right) \rightarrow 0$.

$(2) \Rightarrow(1)$ Let $B^{*}=\left\{x^{*} \mid\left\|x^{*}\right\| \leq 1\right\}$. Suppose a net $\left(x_{\alpha}^{*}\right)$ in $(1 / 2) B^{*}$ converges weak $^{*}$ to $x^{*}$. Then $\left(x_{\alpha}^{*}-x^{*}\right)$ is in $B^{*}$ and for all $(F, \varepsilon)$ it is eventually in $\mathcal{K}(F, \varepsilon)$. Let $g$ be any weak cluster point of $\left(T\left(x_{\alpha}^{*}-x^{*}\right)\right)$. Then $g$ is in $\bigcap_{(F, \varepsilon)} T(K(F, \varepsilon))$, so $g=0$. Thus $T\left(x_{\alpha}^{*}\right) \rightarrow T\left(x^{*}\right)$ weakly in $L^{1}(\lambda)$. It follows that $T$ is weak*-to-weak continuous.

Say that a weakly measurable function $f: \Omega \rightarrow X$ is separable-like provided there exists a separable subspace $D$ of $X$ such that for every $x^{*}$ in $X^{*}$,

$$
x^{*} \chi_{D} f=x^{*} f \text { a.e. }(\lambda) \text {. }
$$

(That is, as far as $x^{*}$ is concerned, $f$ takes almost all its range in $D$.) In particular, simple functions are separable-like. If $(\Omega, \Sigma, \mu)$ is a separable measure space, then every Dunford integrable function is automatically separable-like.

COROLlaRY 4. Suppose $f$ is Dunford integrable and $T$ is weakly compact. If $f$ is separable-like, then it is Pettis integrable.

Proof. Let $\left(x_{n}\right)$ be dense in $D$. Let $g$ be in $\bigcap_{(F, \varepsilon)} T(K(F, \varepsilon))$. We must show that $g=0$ a.e.

For each $n$, choose $x_{n}^{*}$ in $\mathcal{K}\left(\left\{x_{i}\right\}_{i=1}^{n}, 1 / n\right)$ so that $g=x_{n}^{*} f$ a.e. Now choose a fixed null set $E$ so that for every $n, g=x_{n}^{*} f$ off $E$. Let $\left(x_{n}^{*}\right)_{n}$ cluster weak ${ }^{*}$ at $x^{*}$. Then $g=x^{*} f$ off $E$, while $x^{*}=0$ on $D$. Hence,

$$
g=x^{*} f=x^{*} \chi_{D} f=0 \text { a.e. }
$$

If $(\Omega, \Sigma, \mu)$ is a perfect measure space, Geitz [3] shows that every Pettis integrable $f$ is separable-like. Thus, the converse of the Corollary holds for perfect measure spaces.

The next corollary is obvious.

COROLlary 5. Suppose $f$ is Dunford integrable, $T$ is weakly compact, and there is a sequence $\left(f_{n}\right)$ of separable-like integrable functions such that for each $x^{*}$, $\left(x^{*} f_{n}\right)$ converges a.e. to $x^{*} f$. Then $f$ is Pettis integrable. 
If $f: \Omega \rightarrow X$, then the core of $f$ over a set $E$ in $\Sigma$ is defined to be the set

$$
\operatorname{cor}_{E} f=\bigcap\{\overline{\operatorname{co}}(f(E \backslash N)) \mid N \in \Sigma, \lambda(N)=0\} .
$$

LEMMA 6. Suppose $f$ is weakly measurable and that

$$
E \in \Sigma, \quad \lambda(E) \neq 0 \Rightarrow \operatorname{cor}_{E} f \neq \varnothing .
$$

If $x^{*}$ is $X^{*}$, then $x^{*} f=0$ a.e. on $\Omega$ if and only if $x^{*}$ is constantly 0 on $\operatorname{cor}_{\Omega} f$.

PROOF. $(\Rightarrow)$ clearly.

$(\Leftarrow)$ If $x^{*} f$ is not zero a.e., we may assume there exist $E$ in $\Sigma$ and $\alpha>0$ such that $\lambda(E) \neq 0$ and $x^{*} f>\alpha$ on $E$. Then $\operatorname{cor}_{E} f \subset\left\{x \mid x^{*}(x) \geq \alpha\right\}$, and $\varnothing \neq \operatorname{cor}_{E} f \subset \operatorname{cor}_{\Omega} f$, so $x^{*}$ is not constantly zero on $\operatorname{cor}_{\Omega} f$.

Corollary 7 (Geitz-Talagrand). Suppose $f: \Omega \rightarrow X$ is Dunford integrable and $T$ is weakly compact. Then $f$ is Pettis integrable if and only if

$$
E \in \Sigma, \quad \lambda(E) \neq 0 \Rightarrow \operatorname{cor}_{E} f \neq \varnothing .
$$

PROOF. $(\Rightarrow)$ If $f$ is Pettis integrable, then by the separation theorem the integral $\int_{E} f d \lambda$ is in $\operatorname{cor}_{E} f$.

$(\Leftarrow)$ Suppose $(*)$ holds and $g$ is in $\bigcap_{(F, \varepsilon)} T(\mathcal{K}(F, \varepsilon))$, with $g=x^{*} f$ for some $x^{*}$ in $X^{*}$. If $g$ is not identically zero a.e., then there exists $x$ in $\operatorname{cor}_{\Omega} f$ with $x^{*}(x) \neq 0$.

For each $n$, choose $x_{n}^{*}$ in $\mathcal{K}(\{x\}, 1 / n)$ with $g=x_{n}^{*} f$ a.e. Choose a fixed null set $E$ so that for every $n, g=x_{n}^{*} f$ off $E$. Let $y^{*}$ be a weak* cluster point of $\left(x_{n}^{*}\right)$. Then $y^{*} f=g$ a.e., and $y^{*}(x)=0$.

Let $z^{*}=x^{*}-y^{*}$. Then $z^{*} f=0$ a.e. while $z^{*}(x) \neq 0$, contradicting the lemma.

\section{REFERENCES}

1. N. Dunford and J. T. Schwartz, Linear operators. Part I: General theory, Interscience, New York, 1967.

2. D. M. Fremlin and M. Talagrand, $A$ decomposition for additive set-functions, with applications to Pettis integrals and ergodic means, Math. Z. 168 (1979), 117-142.

3. R. F. Geitz, Pettis integration, Proc. Amer. Math. Soc. 82 (1981), 81-86.

4. - Geometry and the Pettis integral, Trans. Amer. Math. Soc. 269 (1982), 535-548.

5. F. D. Sentilles and R. F. Wheeler, Pettis integration via the Stonian transform, Pacific J. Math. 107 (1983), 473-496.

Department of Mathematics, Pennsylvania State University, University PARK, PENNSYlVANIA 16802 